\title{
Ultrasound for Critical Care Physicians: Hypotension
}

A 68 year old man is transferred to the intensive care unit because of hypotension. An ultrasound of the heart and inferior vena cava (IVC) were performed (Figure 1).
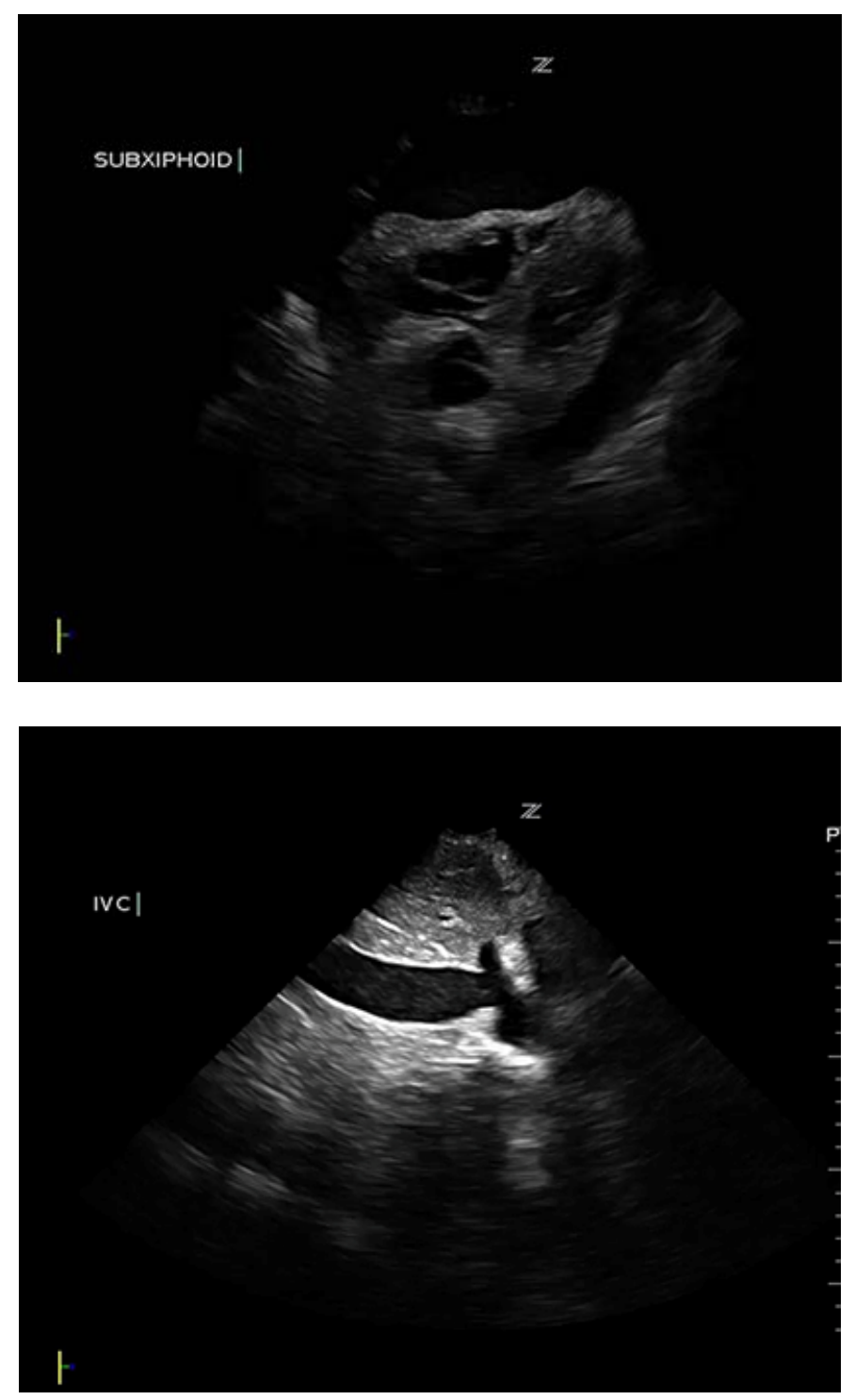

Figure 1. Upper panel: subxiphoid view of heart. Lower panel: inferior vena cava.

What is the cause of the hypotension?

1. Cardiogenic shock secondary to cardiomyopathy

2. Intracardiac thrombus

3. Intravascular volume depletion

4. Massive pulmonary embolism

5. Pericardial effusion 


\section{Correct! \\ 5. Pericardial Effusion}

All the listed disease processes are potential causes of hypotension and ultrasonography can be quite useful (1). The ultrasound subxiphoid view of the heart shows right ventricular collapse and a large posterior pericardial effusion (Figure 2).

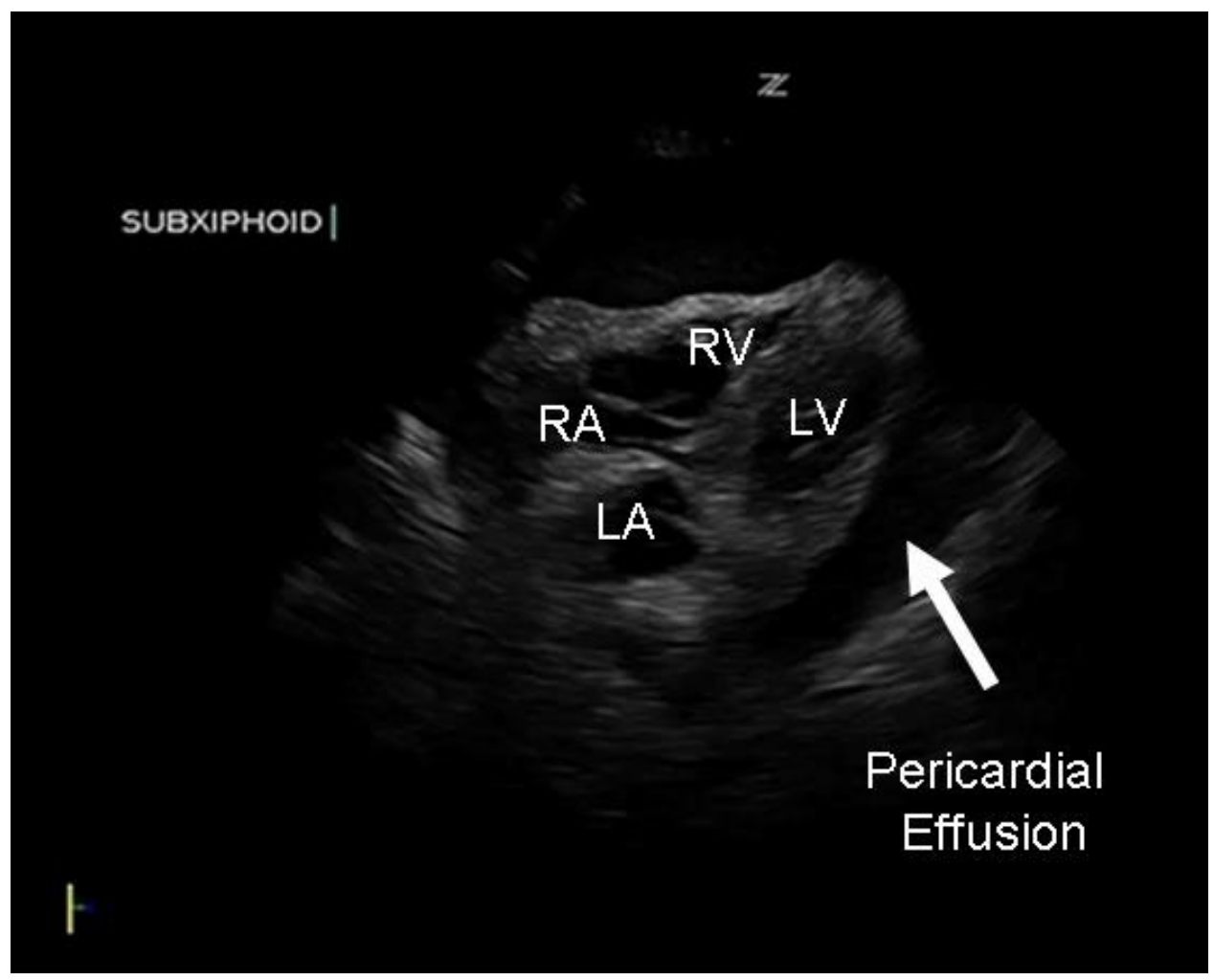

Figure 2. Static image showing the subxiphoid view of the heart with the chambers labeled and a large pericardial effusion.

There is no evidence of an intracardiac thrombus, elevated right sided heart pressures as seen with a massive pulmonary embolism, and the heart muscle is contracting vigorously not hypokinetic as seen with a cardiomyopathy.

The inferior vena cava is greater than $2 \mathrm{~cm}$ (normal 15-17 mm) and does not collapse with inspiration (Figure 3). 


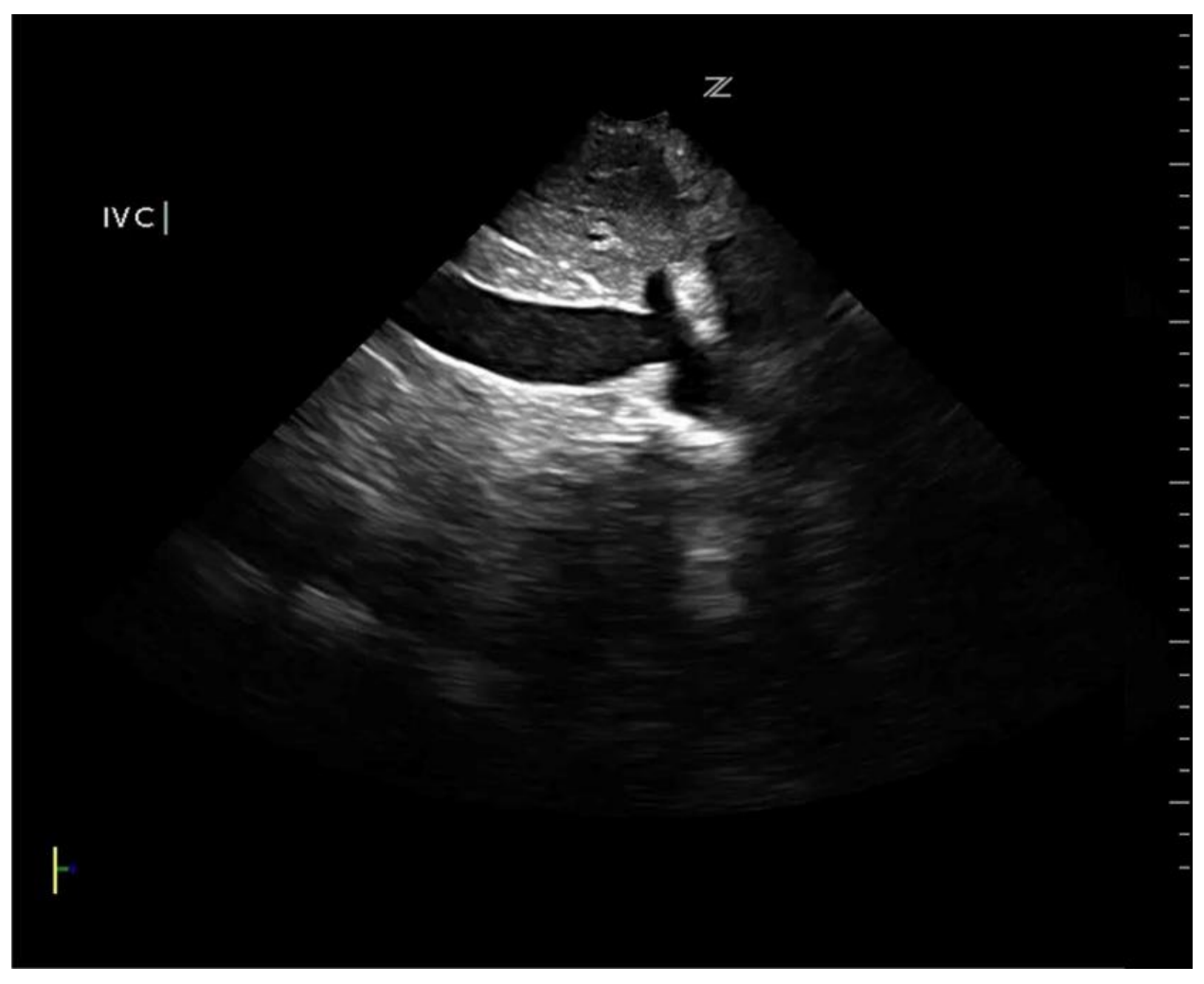

Figure 3. Static view of the enlarged inferior vena cava. Centimeter scale is on the right.

The clinical picture of hypotension with the ultrasonographic findings of a pericardial effusion and an enlarged inferior vena cava suggest the diagnosis of cardiac tamponade.

Jarrod M. Mosier, MD

Department of Emergency Medicine

Department of Medicine, Section of Pulmonary, Critical Care, Allergy and Sleep, University of Arizona, Tucson, Az

\section{Reference}

Atkinson PR, McAuley DJ, Kendall RJ, et al. Abdominal and Cardiac Evaluation with Sonography in Shock (ACES): an approach by emergency physicians for the use of ultrasound in patients with undifferentiated hypotension. Emerg Med J. 2009;26(2):87-91. [CrossRef] [PubMed] 